\title{
Réflexions sur les per diem dans les projets de développement en Afrique
}

Valéry Ridde

\section{(2) OpenEdition \\ Journals}

Édition électronique

URL : http://journals.openedition.org/apad/4111

DOI : $10.4000 /$ apad. 4111

ISSN : 1950-6929

Éditeur

LIT Verlag

Édition imprimée

Date de publication : 1 janvier 2012

Référence électronique

Valéry Ridde, "Réflexions sur les per diem dans les projets de développement en Afrique », Bulletin de I'APAD [En ligne], 34-36 | 2012, mis en ligne le 19 juillet 2013, consulté le 10 décembre 2020. URL http://journals.openedition.org/apad/4111 ; DOI : https://doi.org/10.4000/apad.4111

Ce document a été généré automatiquement le 10 décembre 2020.

Bulletin de l'APAD 


\title{
Réflexions sur les per diem dans les projets de développement en Afrique
}

\author{
Valéry Ridde
}

\section{Introduction}

Avec la quantophrénie aigüe ${ }^{1}$, la perdiemite aigüe est certainement l'une des situations les plus courantes dans les projets de développement. ${ }^{2}$ Lorsqu'un novice de la pratique de l'aide au développement (qu'il soit africain ou occidental) participe pour la première fois à un tel projet, il est immédiatement choqué par cette pratique. Dans le cadre de ses activités, il est rapidement confronté à une question, diplomatiquement posée, à tout le moins en Afrique de l'Ouest « quelles sont les formalités administratives?", "comment motiver le médecin chef? ". D'emblée, le novice est confronté au pouvoir symbolique des mots, qu'il comprendra, plus avancé dans sa carrière de développeur, comme étant aussi les maux de l'aide au développement. Ce qui est également particulièrement frappant dans l'existence de ces per diem est que sa pratique est inversement proportionnelle à sa mise au jour dans les écrits scientifiques. C'est comme si ces maux indicibles, pour reprendre l'expression de Fassin étaient aussi des mots indicibles ${ }^{3}$. Y compris dans le quotidien des projets, la convocation du mot per diem est exceptionnelle, on paraphrase, on élude, on use de litotes car les acteurs sont plutôt dans une logique de "simple modification du vocabulaire afin d'éviter des mots qui effraient» . Mais c'est peut être aussi parce que « on se comprend » et que l'évocation du mot n'est pas nécessaire à l'entendement de la pratique. Un observateur critique des programmes de lutte contre le VIH des années 1980 décrivait la manière dont l'arrivée massive de l'aide et des per diem associés pouvait provoquer une «new epidemic in Africa : Fat Aids» disait-il avec humour, mais dans l'anonymat comme pour témoigner de la difficulté d'avoir un regard 
critique $^{4}$. De plus, rares sont les écrits analytiques sur le sujet alors qu'il ne se passe pas un jour en Afrique où cet objet n'est pas le lieu de discussion, comme cela a été le cas pour l'organisation du colloque de l'APAD en 2010, ou de confrontation entre différents acteurs. Les 300 pages consacrées à un ouvrage visant à "repenser, aujourd'hui, ce que peut être l'anthropologie de l'aide humanitaire et du développement " n'abordent quasiment ${ }^{5}$ pas ce sujet, qui pourtant, lorsque l'on fréquente l'aide humanitaire et le développement, est présent en permanence.

Aujourd'hui, le participant à un atelier de restitution de résultats de recherche, à une formation ou à une action concrète dans le domaine du développement s'attend obligatoirement à ce que l'organisateur lui fournisse une prime, un per diem. Tous les chercheurs et intervenants sont confrontés à ce dilemme éthique où l'octroi d'une somme d'argent incite les personnes à participer. Si les per diem étaient à l'origine prévus pour compenser la perte de temps et d'argent de la participation à l'intervention, ils sont aujourd'hui devenus des instruments politiques qui entachent les activités de recherche et d'intervention dans le monde du développement. Si nombre d'observateurs pensent que l'Afrique n'atteindra pas les Objectifs du Millénaire pour le Développement (OMD) en 2015 , nous croyons que cette question des per diem participe (sans tout expliquer évidemment) à l'échec prévu, car ils réduisent l'efficacité potentielle des interventions. L'objet de ce texte n'est évidemment pas de jeter la pierre sur quiconque, car nous verrons combien le sujet est complexe et les complicités nombreuses. Il s'agit plutôt d'essayer d'en noter l'histoire, de comprendre les causes, d'en relever les pratiques et les conséquences et de formuler quelques pistes de solutions de manière à ce que ce phénomène trouve enfin un traitement équitable. Notre proximité des projets de développement ${ }^{6}$ dans le domaine de la santé nous fournira la majeure partie des exemples empiriques. De plus, pour beaucoup d'acteurs rencontrés, le secteur de la santé semble être le parangon du déploiement des per diem. En effet, si nous avons retrouvé quelques rares articles qui abordent la problématique des per diem dans différents secteurs de la solidarité internationale, dont la construction de logements, le développement économique ou encore l'approvisionnement en eau , il faut reconnaître que le secteur de la santé est à ce sujet le thème le plus fréquemment associé aux pratiques de per $\operatorname{diem}^{7}$. Mais revenons à la définition de notre objet de discussion.

\section{De quoi parle-t-on?}

3 Lorsque l'on tente de lire les écrits scientifiques du développement à l'aune de l'usage des per diem, deux constatations s'imposent : la rareté dont nous avons déjà parlé et la confusion des termes. Les pratiques sont tellement disparates et nombreuses qu'il ne semble pas exister, dans la terminologie en langue française comme en anglais, de terme univoque pour parler de ces sommes monétaires. On parle indifféremment d'indemnité, de prime, ou de per diem en français; et de "allowances", "incentive» ou "perdiem» en anglais. Mais ces termes sont également utilisés pour qualifier des sommes d'argent données à d'autres occasions, comme les primes à la performance, les indemnités complémentaires de salaires, et autre vocabulaire des promoteurs du New Public Management. Ainsi, aucune définition n'a été trouvée dans notre parcours de la littérature sur le 
développement. La seule tentative de définir le terme « per diem » remonte à 2003 et n'est pas directement liée à l'industrie du développement. Dans son journal Terminologie comptable, le Comité de terminologie française de l'Ordre des comptables agrées du Québec propose deux définitions :

-«somme accordée sur une base journalière pour compenser les frais de subsistance engagés à l'occasion de déplacements hors du lieu habituel de travail »;

5 - « rémunération calculée à la journée pour la participation à des réunions ou pour l'exécution d'un mandat à durée déterminée "

Les terminologies françaises correctes pour ce Comité concernant les deux définitions précédentes seraient respectivement « indemnité journalière » et « tarif journalier ». Mais si nous revenons au contexte plus spécifique des projets de développement, l'étude des écrits montre que le terme "per diem " recouvre plusieurs réalités qui se confondent la plupart du temps,:

7 -dédommagement (pour compenser des frais de subsistance engagés à l'occasion d'un déplacement en dehors du lieu principal de travail) ;

10 Dans cet article, nous mettrons pour le moment de côté cette troisième forme car elle est encore trop récente en Afrique pour être l'objet d'une étude spécifique et rares sont les expériences qui ont été l'objet d'une analyse sérieuse non biaisée par des idéaux et présupposés comme cela est parfois le cas .

\section{De l'arrivée des per diem et de l'ordre négocié entre développeurs}

11 Sans avoir la prétention ici d'écrire l'histoire des per diem pour l'Afrique, nos discussions avec plusieurs anciens cadres de ministères et coopérants nous laissent croire que ces pratiques de per diem sont apparues à la fin des années 1970 concomitamment à l'accroissement de la mise en œuvre des projets de coopération internationale. Jusqu'à cette date, les agents du système de santé réalisaient leurs activités en étant rétribués par leur salaire mensuel de fonctionnaires, sans autre rétribution et indemnité que les frais de déplacement. Ils étaient souvent accueillis dans les provinces par leurs collègues ou les villageois qui les logeaient et les nourrissaient simplement. À partir de ce moment, il semble que ce soit surtout l'arrivée massive de "l'industrie » de la coopération internationale qui a provoqué l'organisation de ces modalités de financement. Un ancien coopérant se souvient du discours du nouvel ambassadeur étatsunien, novice en Afrique comme le reste de la coopération américaine, affirmant qu'il allait déployer de nombreux projets et faire « des paysans maliens de petits bourgeois ». L'idéologie derrière la mise en œuvre des projets était fondée sur une motivation largement financière. Les êtres humains ne travailleraient que pour de l'argent. L'argent va pouvoir les motiver, tel qu'en témoigne aujourd'hui le discours employé pour justifier les per diem car "il faut les motiver" entend-on souvent. C'est la même justification qui est aujourd'hui utilisée par les promoteurs des contrats de performance pour fournir 
les services de santé, l'idéologie de la performance disent certains. Or la recherche, notamment les premiers travaux de Karasek dans les années 1970, a montré depuis bien longtemps que si la rétribution fait partie des sources de motivation, elle ne saurait en être l'unique. Mais cette approche fondée sur l'argent n'est pas spécifique à la coopération nordaméricaine.

Dans un autre pays africain, un ancien cadre du ministère de la santé nous disait que les projets ont multiplié la présence de coopérants grassement payés. Pour leurs projets, ces individus réalisaient des activités avec leurs collègues nationaux bien moins payés qu'eux. Aussi, ils ont souhaité introduire ces per diem, peut être par souci éthique, mais surtout par désir d'efficacité ${ }^{8}$ afin que les activités puissent avoir lieu et qu'ils montrent à leurs responsables que le projet avance (voir supra). Il s'agit d'un des symptômes du " project mentality ". Nous sommes là au cœur de l'ordre négocié des projets de développement où l'on doit mettre de côté les choses qui fâchent pour avancer. Nous y reviendrons dans notre discussion des conséquences de ces pratiques. De plus, les experts nationaux, "spectateurs du train de vie des experts", commençaient aussi à se plaindre de ces écarts aberrants de salaires avec les coopérants et les experts internationaux en mission. Un africain que nous interrogions sur l'histoire se souvient d'une évaluation à laquelle il a participé comme expert national avec un européen "Moi, mon salaire là, représentait le perdiem de 5 jours » de cet expatrié, même si évidemment le premier ne faisait cette consultation ni bénévolement ni dans le cadre de son travail'. Fournissons un autre exemple présenté par un collègue. Un médecin d'un pays d'Afrique de l'Ouest, de retour d'une spécialisation en santé publique en Belgique, gagne un salaire mensuel de 200.000 F CFA (300 Euros) dans son ministère alors que son collègue médecin européen ayant le même diplôme de spécialité dans la même université gagnera au moins 3.000 Euros en étant contractuel d'un projet de coopération. Puis, au fur et à mesure des années et des décennies du développement, les habitudes ont été prises. Ce fonctionnement est entré dans les mœurs. Il est aujourd'hui devenu quasiment un droit. Il devient maintenant impossible de faire quoique ce soit sans ces per diem dont une grande partie des causes peuvent être maintenant décrites à grands traits.

\section{De quelques causes}

\section{La mise en œuvre des projets}

13 Par la distribution de ces ressources monétaires, on cherche moins à faire en sorte que le projet soit efficace en produisant des effets pour les populations que de s'organiser pour que les activités soient mises en œuvre, dans un but évident de produire des rapports aux bailleurs de fonds sur la réalisation des activités et de justifier la présence de ceux qui les organisent ${ }^{10}$. Or, les activités des projets ne se déroulent pas dans le néant. Elles s'adressent à des personnes qui ont déjà des activités professionnelles ou sociales. Le paysan cultive son champ, l'infirmier vaccine les enfants, l'instituteur enseigne les mathématiques et le fonctionnaire de l'administration centrale écrit des rapports. Aussi, les projets sont-ils en concurrence entre eux mais aussi en concurrence avec les activités régulières des 
personnes auxquelles ils s'adressent. Le per diem joue donc dans ce cas un rôle incitatif pour intéresser les acteurs aux activités du projet.

Projets et concurrence : Aussi, des responsables de projets vont proposer des taux journaliers plus importants qu'un projet concurrent pour être certain qu'ils auront plus de fonctionnaires à leur formation. Malgré la fameuse déclaration de Paris, les projets et autres bailleurs de fonds n'arrivent pas toujours à s'entendre. Au Burkina Faso comme au Niger, le gouvernement a voulu légiférer sur ce sujet afin de mieux réguler les pratiques. En 2007 au Burkina, cinq décrets présidentiels ont porté sur le fonctionnement des projets. À cette occasion, l'organisation des per diem et autres primes (logements, sujétion, etc.) a été précisée. Des montants précis ont été décrétés selon les catégories de personnes et activités concernées. La hiérarchie des per diem a été consacrée, nous y reviendrons. Mais ce qu'il faut ici retenir est que les bailleurs de fonds, n'ont, d'une part, pas tous souhaité s'aligner (pour reprendre un des termes de la déclaration de Paris) sur ces montants, et, d'autre part, ne se sont pas entendus entre eux pour harmoniser l'alternative. Certains suivent ces consignes à la lettre, d'autres les adaptent, d'autres utilisent une autre grille gouvernementale (ce qui montre les problèmes de coordination au sein même de l'État ${ }^{11}$ ) et d'autres enfin disposent de leur propre système. Les pays européens, la Suisse et le Canada ont répondu à ces décrets en envoyant une lettre au ministre de l'économie et des finances en avril 2008 pour le féliciter de cette décision. Mais ils affirment que "plusieurs éléments ou niveaux de rémunérations et indemnisations qui ne peuvent être applicables en l'état, n'étant pas cohérents avec les règlements et accord de coopération en vigueur ». Face à la « sensibilité de la question et de la difficulté à la gérer ", ils proposent que ces changements financiers soient pris en charge «par le budget national » notamment dans le cadre de la contrepartie nationale exigée dans la plupart des projets. Démarche osée, mais qui n'aura pas de suite car le ministre n'a pas répondu à la lettre et n'a pas donné suite à ce sujet, si ce n'est dans une discussion de couloir avec certains partenaires lors d'une réunion.

Les pays donateurs de l'aide au Burkina Faso n'ont pas réussi à coordonner leurs pratiques. De multiples systèmes fonctionnent. Par exemple, un responsable d'une agence de coopération européenne nous dit ne donner aucun per diem lorsqu'il s'agit de rencontres ou d'ateliers qui ne donnent pas lieu à des déplacements. Il faut en effet reconnaître qu'une telle distribution monétaire ne correspond plus au défraiement des sommes engagées pour participer à une réunion mais bien à une rémunération qui ne dit pas son nom. L'article 4 du décret 779 de 2007 tente de légaliser cette situation en précisant que «la participation à des séminaires et ateliers de formation n'impliquant pas de déplacement du participant en dehors de son lieu de résidence, donne droit au versement d'un montant de 5000 (cinq mille) francs par jour». Bien que ce responsable nous dise que cela ne pose pas de problème particulier, une personne d'une autre agence nous dira " ils ont beaucoup de problèmes, les burkinabè les boycottent ». Dans une rencontre à 300 $\mathrm{km}$ de la capitale avec un agent d'un projet financé par cette même coopération, nous apprenons que ce principe n'est pas toujours respecté. Les hauts commissaires et autres gouverneurs ne semblent pas prêts à participer à un atelier organisé dans leur localité sans per diem, même s'ils sont nourris et n'ont pas à se déplacer d'une localité à une autre. La même logique est rencontrée au Mali, où les 
agences des Nations Unies ont décidé en 2010 que les fonctionnaires de l'État résidents dans la capitale et suivant une formation dans cette même ville devaient percevoir $5.000 \mathrm{~F}$ de frais de déplacement.

\section{Projets et paix sociale}

16 D'autres projets vont "courir " après un fonctionnaire pour lui remettre le per diem que ce dernier a refusé (en jouant consciemment sur ce pouvoir), car ils veulent que leur projet réussisse, ou plutôt, que les activités soient mises en œuvre avons-nous dit plus haut. Un responsable expatrié de projet nous confiait « on ne va pas lui dire que ce n'est pas bien sinon il va tout bloquer et on ne pourra pas avancer ». Cette même personne, lors de la journée de présentation des résultats de l'évaluation de son projet décide de donner un taux de per diem plus haut que d'habitude pour les participants à l'atelier afin "d'avoir la paix ", nous dit-il. Il s'assure ainsi du peu de regard critique des parties prenantes. Le responsable de cette coopération européenne qui refuse de payer la participation à un atelier avoue " pour ne pas être en conflit avec les partenaires nationaux, on ne parle pas de cela ». On voit bien là le caractère " sensible » de la question, tel que le mot a été employé par le chef de la délégation européenne dans sa lettre précédemment citée. Cette pratique n'est pas l'apanage des expatriés en particulier mais plutôt des développeurs en général. Une jeune animatrice dans un projet de développement nous avouera avoir été stupéfaite par ces pratiques lorsqu'elle a démarré sa carrière. Mais, tout comme les élèves sages-femmes qui avaient appris les bonnes pratiques à l'école se voient dans l'obligation d'user de mauvaises pratiques une fois dans le contexte de leur service, cette animatrice raconte « on paye pour avoir la paix ». Le terme de paix signifie dans ce contexte, la volonté de ne pas créer des conflits dans le but évident de réaliser les activités des projets conformément aux prévisions.

\section{Disposer de revenus supplémentaires}

17 Les programmes d'ajustement structurel, l'imposition du maintien voire de la réduction de la masse salariale et l'affaissement du rôle des États en Afrique ont fait que ces per diem sont devenus vitaux pour les fonctionnaires. Ils sont un moyen de compenser une activité faiblement rémunératrice dans des pays caractérisés par des niveaux de vie très bas car la plupart du temps «le montant accordé sera bien supérieur aux dépenses réelles que l'apprenant aura à engager » dans le cas de formation par exemple. Ils sont progressivement devenus des sources de revenus complémentaires qui ne rentrent évidemment jamais dans l'assiette fiscale et le calcul des impôts sur le revenu. Ainsi, la possibilité de percevoir des per diem pour la participation à des activités en dehors du lieu de travail est une des raisons avancées par les participants d'une étude menée au Cameroun pour justifier qu'ils préfèrent garder leur travail dans le secteur public malgré un salaire faible, dans un contexte de crise économique. D'autres études donnent une estimation de la proportion des revenus qui peut être attribuée aux per diem ${ }^{12}$. Ainsi au Ghana, cette proportion va de $36 \%$ pour les assistants médicaux à $80 \%$ pour les médecins . Au Malawi, une étude indique qu'une formation de cinq jours permet à une infirmière d'augmenter de 25 à $40 \%$ ses revenus mensuels . 
McCoy et ses collègues montrent, quant à eux, que $10 \%$ environ du salaire du personnel de santé au Burkina Faso est attribuable aux per diem . Au Burkina, une étude précise que les agents de santé s'octroient en moyenne 30\% de ristournes sur les actes médicaux alors que la norme officielle est de $20 \%$. Cette étude sous-estime certainement ce pourcentage car elle est basée sur la déclaration des personnes et ne concerne pas les médecins ${ }^{13}$ qui sont souvent les plus concernés par les réunions de formation et autres ateliers de validation. Dans la même perspective, une étude menée au Vietnam montrent que les participants interrogés considèrent la participation aux formations comme une activité génératrice de revenus à part entière . Certains ont même inventé un drôle (et néanmoins vulgaire) de néologisme lorsque le taux accordé est trop faible et qu'il ne correspond pas à leurs attentes : un «merdiem ». Mais cette recherche de revenus supplémentaires n'est pas cantonnée aux cadres ou au domaine de la santé, un jeune chercheur possédant un doctorat démarre sa carrière avec moins de $200.000 \mathrm{~F}$ CFA par mois ). Un chauffeur bénévole dans un district nous avoue profiter des missions pour disposer de revenus stables qu'il ne peut avoir puisqu'il n'est pas fonctionnaire. En un an, il nous explique qu'il a réussi à faire « une maison en banco. Ça m'a couté au moins plus de $600.000 \mathrm{~F}$ », ce qui à l'aune de la richesse au Burkina n'est pas une petite somme (voir note 13). Mais en discutant un peu plus avec lui on comprend qu'il s'organise aussi pour faire des réparations mécaniques pour les districts de la région en fournissant des fausses factures produites dans le garage où il a fait son apprentissage et en donnant $2.500 \mathrm{~F}$ par facture au petit frère du garagiste car ce dernier semble réticent à cette pratique.

Si les agents de santé en profitent, les populations ne sont pas aveugles, elles connaissent parfaitement ce mode de fonctionnement. Une femme d'un village nous disait à propos des infirmiers qui viennent depuis quelques années soigner dans le dispensaire de leur village : "Si on leur refuse ces per diem, ils ne s'intégreront pas aux problèmes du village. S'ils ne doivent compter que sur leur salaire, si le mois est avancé, ils connaîtront un jour des problèmes et ne seront pas à l'écoute des populations $»^{14}$.

\section{Désir d'ostentation, rôle social et train de vie}

19 Un autre facteur pouvant expliquer l'institutionnalisation des per diem, et la course effrénée aux formations et aux réunions qu'elles engendrent, est la nécessité d'assumer son rôle social, tel que la société s'y attend ou tel que l'individu souhaite le voir. Jaffré explique ainsi qu'en Afrique de l'Ouest, les salaires trop bas des personnels de santé ne leur permettent pas d'assumer les échanges intercommunautaires sur lesquels se fonde l'entraide entre familles ou promotionnaires. Ne pas se conformer à ce code de la générosité revient alors à prendre un véritable «risque social» de marginalisation. Le revenu supplémentaire que fournissent les per diem constitue donc un moyen informel d'assumer son rôle social. Le per diem devient ainsi un objet de compétition et un instrument de pouvoir. En effet, comme le déplorent également Muula et Maseko , les opportunités de formation et de réunions sont monopolisées par les cadres au détriment des professionnels à qui elles seraient réellement profitables. Ce caractère très inégalitaire de la distribution des per diem sera discuté plus loin. 
Mais il faut aussi relever que l'on risque aussi la marginalisation si l'on ne rentre pas dans cette logique sociale propre aux projets. Un membre d'une institution de recherche nous explique qu'à son retour de mission, n'ayant pas utilisé toute la somme fournie au départ, car calculée selon des normes UEMOA bien au-delà des besoins réels, décide de remettre le solde au comptable. Quelle ne fût pas la surprise du comptable et surtout sa réaction de réprobation d'un tel comportement qui pouvait mettre à mal des droits acquis de longue date. Contrainte et forcée, cette personne a du conserver les sommes non utilisées. Même pour les per diem, il est difficile de s'écarter des normes pratiques, qui plus est, avec de l'argent d'une institution africaine et non occidentale, ce qui montre bien que la fameuse dichotomie Nord/Sud ne fonctionne pas pour cette logique.

En effet, on aura également bien compris que le problème n'est pas du seul ressort des habitants des pays à faible revenu. Nombres d'expatriés, d'experts internationaux et de chercheurs des pays riches en profitent aussi allègrement. Les taux de per diem pour leurs déplacements dépassent souvent largement le coût de la vie dans les pays qu'ils visitent, et on ne parlera pas ici des primes d'expatriation octroyées par certaines institutions. Alors que la vie dans un hôtel moyen d'une capitale ouest-africaine peut leur coûter en moyenne 50.000 F CFA, ils vont recevoir un per diem d'au minimum de $100.000 \mathrm{~F}$ CFA. Une mission à Dakar pour une agence des Nations Unies, tel que l'auteur de cet article a pu aussi en bénéficier ${ }^{15}$, fournit un per diem quotidien de $300 \$$ US, soit presque $150.000 \mathrm{~F}$ CFA ou encore 1,6 fois le seuil de pauvreté annuel au Burkina Faso. Mais comme le disait une canadienne que nous interrogions et qui était critiquée par ses collègues de s'organiser plus de missions à l'étranger que nécessaire, et de vivre dans des conditions précaires lors de ces déplacements, «cela me permet de faire des économies». En effet, chacun sait, qu'il soit occidental ou africain, que tout le monde réalise des économies sur les per diem, soit car ils sont au dessus des besoins réels, soit simplement par des choix stratégiques où l'on ira dormir dans un hôtel le moins cher possible (les fameuses cases de passage) ou tout simplement chez un parent. Une animatrice nous dira même qu'elle ne mange qu'un fruit le soir tant les pauses café et les repas fournis lors des ateliers sont largement suffisants.

\section{De quelques conséquences et effets pervers}

21 Si les per diem sont parfois reconnus comme un facteur de motivation, au même titre que les avantages monétaires, parmi lesquels "on-call allowance", "mountain allowance», "travel allowance", «housing allowance", « risk allowance ", ou "medical allowance», ils sont davantage reconnus comme une pratique néfaste pour les projets de développement. En même temps, ils participent à maintenir un statu quo, à entretenir un ordre négocié et des relations de clientélisme et de pouvoir discrétionnaire, selon des logiques bien connues dans ce milieu .

\section{La course au per diem et les stratégies de capture}

22 D'abord, considérant l'apport financier non négligeable que les per diem rapportent, on assiste à une véritable «course au per diem ", pour reprendre le 
titre d'un article d'un journaliste burundais . L'auteur n'hésite pas à qualifier cette course de "sport national ». Au Nord Bénin certains ont utilisé le terme de " chasse au per diem » . L'objectif est donc de multiplier sa présence au plus grand nombre de formations, ateliers et réunions possibles proposant des per diem, parfois même au cours de la même journée. Les participants ont ainsi développé un véritable don d'ubiquité, don qui se réalise dans la pratique répandue du saut de grenouille («frog-leap») . Cette stratégie consiste à signer les feuilles de présence de plusieurs réunions au cours d'une même journée sans y assister réellement, comme l'explique un professionnel de la santé du Malawi : «What you have to do is just be available for sometime, register for the workshop and move on to the next workshop that you have been invited. In so doing, you are assured of the allowances. " Swidler et Watkins évoquent de leur côté une nouvelle forme de " chasse-cueillette» (" hunting and gathering ») qui vient compléter les maigres moyens de subsistance de la communauté, surtout parmi les volontaires qui n'ont pas d'autres sources de revenus et qui sont de fervents adeptes de ces rencontres. D'autres tactiques frauduleuses sont citées par les deux auteurs, comme la surfacturation des frais d'essence, ou l'utilisation des véhicules de service, mais c'est un autre sujet. Lors de nos propres observations, nous avons vu également certains signer à la place d'un collègue absent pour obtenir et se partager les sommes par la suite. On s'organise aussi pour que des réunions prévues pour la journée entière se termine à 14 heures, afin de toucher le per diem de la journée entière et reprendre d'autres activités. La même stratégie est déployée pour raccourcir un atelier de trois jours en deux jours, en travaillant un peu plus les deux premiers jours mais pour toucher par la suite les trois jours dans leur totalité. Réduire la durée d'un atelier est quasiment impossible si le montant total des per diem n'est pas maintenu. Alors que les organisateurs d'une réunion souhaitaient faire un tel raccourci, une dame au fonds de la salle s'est levée pour dire " on a déjà pris des engagements ». Le novice en per diem ne comprendra pas. Mais celui qui navigue dans ces sphères depuis longtemps saura que cette personne a voulu dire qu'elle avait d'ores et déjà prévu des dépenses (ou contracter un prêt) équivalent à la totalité de la somme prévue. La réduire est évidemment impossible. Aussi, quasiment plus aucune activité ne peut être réalisée sans ces per diem. Rares sont les agents de santé qui iront vacciner les enfants s'ils ne "gagnent pas quelque chose " comme lors des Journées Nationales de Vaccination. Jaffré résume parfaitement la réalité derrière ces pratiques pour les professionnels de santé : « être un bon père ou chef de famille implique d'être un soignant cupide $»^{16}$.

\section{De la corruption légalisée}

23 "C'est de la corruption légalisée", voici comment un expatrié rencontré pour discuter de cette pratique qualifie les per diem. Après une heure de discussion sur le sujet, nous nous quittons. Mais quelques minutes après, il nous envoie un message par téléphone, qu'il répètera quatre fois le lendemain, pour nous demander de ne pas du tout évoquer les détails de notre entretien alors même que nous avions été d'accord sur le caractère anonyme (non pas confidentiel) de notre discussion. Ce comportement nous paraît consubstantiel à cette pratique des per diem, le dénoncer comme étant de la corruption lors d'un colloque intime, mais ne 
surtout pas vouloir porter le débat au grand jour et scier la branche sur laquelle nous sommes assis. Toujours est-il que les pratiques de per diem constituent des enjeux financiers et politiques importants, illustrés par la mainmise des cadres sur ces sources de revenus. Autour de ces enjeux se sont instaurées des pratiques de corruption et de clientélisme, comme le montre une étude menée dans un quartier de Cape Town en Afrique du Sud . Afin d'assurer la participation des résidents aux réunions pour développer des projets de construction, des per diem ont été mis en place. Cette pratique a été détournée pour servir les intérêts du comité de projet qui favorisait la participation des partisans d'un parti politique sud-africain. Kamoche rappelle également que les avantages financiers accordés au cours des formations sont l'apanage de ceux qui maîtrisent les rouages de l'aide au développement et qui profitent pour en faire bénéficier leurs amis. Ces pratiques atteignent leur apogée quand il s'agit d'indemniser des cadres pour obtenir leur bénédiction, comme en témoigne un assistant de santé au Malawi : "The DHO [district health officer] is the overall in-charge of the district. Although s/he may not be physically at the workshop, he is spiritually with you and so $\mathrm{s} /$ he deserves the allowances ". Dans un pays Sahélien, un projet italien a décidé d'octroyer une prime mensuelle au directeur régional afin qu'il soit le «point focal » du projet, autre terme à la mode dans le développement, alors même qu'il n'intervient absolument pas dans les activités de ce dernier. Lors de l'évaluation finale, les consultants, qui ont en vain cherché à le rencontrer, ont compris que son indisponibilité était assurément liée à cette prise en charge. La limite entre la corruption ${ }^{17}$ et le dédommagement est donc tenue.

\section{S'éloigner plus pour gagner plus}

Dans certains pays, les taux de prise en charge sont plus importants en dehors de la capitale que dans celle-ci car les déplacements occasionnent une hausse des per diem. Ainsi, il n'est pas rare de voir que l'atelier est organisé dans un lieu à 35/45 $\mathrm{km}$ de la capitale, considéré comme périphérique, pour obtenir un taux plus élevé mais en même temps permettre à tout le monde de rentrer le soir à la maison. Selon les pays, certains sites sont largement connus de tout le monde pour leur capacité d'accueil de telles rencontres. Un programme national dans le domaine de la santé organise même toute une logistique pour que cela se passe bien et que les participants puissent rentrer avec suffisamment d'argent. Une de ces participantes nous raconte qu'on lui a octroyé $25.000 \mathrm{~F}$ (taux prévu si déplacement) pour sa journée d'atelier passée à moins d'une heure de la capitale, alors que les repas étaient servis et qu'un bus a transporté les participants vers le lieu de la rencontre. Au Sénégal, l'existence de la mer fait en sorte que des hôtels en bord de mer un peu éloignés de Dakar profitent assurément de cette rente pour le développement de l'industrie hôtelière. Car il faut bien aussi reconnaître que les per diem profitent aussi à toute une économie. La présence des vendeurs de pagnes n'est-elle pas consubstantielle à celle des participants à des ateliers ?

\section{Trainingism et absentéisme}

Devant l'enjeu financier et politique que constituent les per diem, les institutions, les cadres et décideurs, qui bénéficient également de ces pratiques, ont un intérêt 
certain à maintenir, voire à favoriser, la culture de la formation. Ce statu quo ne fait qu'entretenir le "workshop syndrome», cette profusion de réunions, rencontres et ateliers qui caractérise le quotidien des agences d'aide internationale. D'autres, vont nommer cette pratique le «trainingism », qui reflète la nécessaire justification des formations afin de maintenir les avantages afférents ${ }^{18}$. Dans une étude réalisée auprès de médecins provenant de 10 pays africains, ces derniers avouent que la stratégie la plus couramment déployée selon eux pour obtenir des subsides supplémentaires étaient la participation à des ateliers .

26 Qui n'a pas constaté qu'en fin d'année budgétaire les activités se multiplient par miracle, et notamment les formations dont la pertinence est souvent loin d'être convaincante? Des responsables vont envoyer leurs subalternes dans des formations, parfois pour une quatrième fois sur le même thème. En effet, ce sont souvent " les mêmes qui sont proposés ou qui se proposent ». Par exemple, un infirmier d'un district que nous visitions affichait sur sa porte le nombre de formations suivies: soit 13 formations en cinq ans pour une durée totale de 82 jours. Un témoignage au Burundi confirme: «Il arrive que des fonctionnaires suivent cinq formations identiques. Au bout du compte, ils n'ont rien appris » . Les intervenants planifient des actions dans le but principal de bénéficier de per diem et non pour impulser un changement auprès du public cible de leur intervention. D'ailleurs, toutes les connaissances acquises et les changements de pratiques des participants aux multiples formations ne sont que très rarement évaluées. Quels sont les formateurs qui, de manière systématique, demandent aux participants de remplir un questionnaire avant et après la formation pour en évaluer les acquis à court terme? Dans un chapitre de réflexions sur le sujet, Gruénais s'interrogeait fort justement «on est parfois amené à se demander si la seule finalité des formations n'est pas la formation elle-même ».

Que reste-t-il finalement aux participants des formations? Entre ceux qui ne sont pas réellement présents, comme ce fonctionnaire qui avoue « passer juste avant la pause café du matin » pour signer la feuille de présence et revenir à la fin des activités pour percevoir son dû , et ceux qui n'ont aucun intérêt professionnel à assister à la formation mais qui s'y rendent pour toucher les per diem, on peut légitimement s'interroger. McCourt et Awases considèrent ainsi que le modèle de développement basé sur la formation et le développement des compétences est tombé en disgrâce en partie à cause des intérêts nichés dans ces activités. Nombre de bailleurs de fonds souhaitent depuis longtemps, sans vraiment y parvenir, réduire leur contribution financière à ces activités. Il est parfois difficile de clamer publiquement que l'on est contre le renforcement des capacités, en tous les cas, tel qu'il se pratique bien souvent. Comme pour le paiement de la participation aux ateliers, celui qui concerne les formations a aussi changé. Un ancien responsable du domaine de l'éducation au Burkina nous rappelle l'histoire des groupes d'appui pédagogique. Lorsqu'ils ont été créés pour favoriser les discussions pédagogiques entre enseignants, ces derniers se cotisaient pour que les rencontres puissent avoir lieu. Par la suite, leur présence devait donner lieu à des repas pour favoriser leur participation, ce qui plus tard s'est transformé en distribution obligatoire de per diem. Aujourd'hui, le ministère n'ayant plus les ressources pour les soutenir, ces groupes d'appui ne se réunissent plus. La même situation vient de se passer dans le 
domaine de la santé où les comités techniques de préparation du Plan national de développement sanitaire (PNDS) 2011-2020 n'ont pas pu se réunir en décembre 2009 , le budget per diem ayant été épuisé. L'organisation d'une conférence nationale sur les politiques de gratuité des soins au Niger en 2012 a longtemps été bloquée par l'absence de per diem suffisants et la multiplicité des commissions et sous commissions souhaitées; " cette question de perdiem a trop duré et moi, je suis sous pression des membres du comité » disait un responsable. La première liste des personnes invitées contenait plus de 250 personnes. On peut en effet comprendre cette difficulté à mobiliser des fonds qui parfois sont énormes. La planification de la révision de la politique sanitaire nationale du Burkina et de la formulation du PNDS 2011-2020 devait coûter, selon le budget envoyé aux partenaires techniques et financiers en juin 2010, plus de 160 millions de F CFA, dont $50 \%$ pour les per diem consacrés aux divers ateliers (120.000 Euros), sans compter les pauses repas et autre indemnités pour les membres du comité technique d'élaboration. On ne saura pas s'il s'agit d'une conséquence de ces abus ou une véritable prise de conscience, mais l'État a décidé pour l'exécution du budget 2012, « la suppression de la perception de perdiems non prévus par un texte réglementaire ». Le conseil des ministres venait en effet, sur la base d'un rapport circonstancié, prendre conscience de l'« absence de base réglementaire ainsi que des abus et risques pour la gestion des ressources financières de l'Etat et de ses démembrements... ». Le rapport affirme notamment qu'en 2011, trois $^{19}(50 \%)$ des six milliards de F CFA engagé pour des activités d'ateliers, de conférences et autres formations étaient consacrés à la prise en charge des participants. Ainsi « le conseil a instruit le ministre en charge des Finances de prendre des mesures idoines pour mettre fin aux dérives de gestion liées à la pratique des per diem. Seuls les paiements autorisés par les textes en vigueur seront désormais servis ».

Découlant directement de cette course effrénée aux per diem, l'absentéisme est un autre effet pervers. Quand le personnel est en formation ou en réunion, cela signifie qu'il n'est pas sur son lieu de travail, à assumer les responsabilités pour lesquelles il est rémunéré. Dans les années 1990, une étude en Tanzanie révèle que, sur une période de quatre mois, l'équipe régionale de la santé passait $40 \%$ de son temps à l'extérieur de la région, le plus souvent pour assister à des séminaires. Une étude au Burkina Faso a montré que dans sept districts ruraux, le taux d'absentéisme des médecins était de $15 \%$ à $65 \%$ selon les districts . L'absence prolongée de ces participants implique non seulement une charge de travail supplémentaire pour le personnel restant, mais également une faible performance des ressources humaines. Dans des pays qui subissent une crise des ressources humaines, notamment dans le secteur de la santé, ces "pertes indirectes" s'apparentent à un véritable gaspillage .

\section{Renforcer les inégalités et le népotisme}

La distribution des per diem vient souvent confirmer et renforcer les hiérarchies en place et les inégalités de traitement. En effet, la plupart des barèmes de per diem suivent la règle administrative (et sociale) de la hiérarchie qui est évidemment souvent aussi source de difficulté, car "plus tu montes dans la hiérarchie plus c'est un problème » nous dit-on. Ainsi, le chef de service touchera 
un montant supérieur au chauffeur qui l'a conduit vers le lieu de la réunion. Encore une fois, on voit bien là que l'origine du per diem qui visait à défrayer les participants a été détournée pour se transformer en une rémunération cachée qui suit la pyramide salariale...et sociale. Comment en effet justifier autrement que par cette logique hiérarchique que le chauffeur dispose de besoin alimentaire ou de logement différent que son chef de service ? L'une des explications fournies par un cadre d'un projet de développement et ancré dans cette vision hiérarchique de la vie est qu'il existe " des pensants et des non pensants ». Il ne s'agit donc plus de dédommagement mais bien d'une rémunération suivant une échelle salariale. Les décrets présidentiels du Burkina Faso tout comme la quasi-totalité des projets entérinent également cette disposition. Les frais de mission à l'intérieur du pays dans un chef lieu de région donnant lieu à une nuitée sont payés à $25.000 \mathrm{~F}$ pour le personnel de catégorie 1 et à $10.000 \mathrm{~F}$ pour le personnel de la catégorie 4 et 5 . Au Niger, le décret de $2007^{20}$ précise que les indemnités journalières pour frais de déplacement à l'intérieur du pays sont de $25.000 \mathrm{~F}$ CFA pour les fonctionnaires de catégorie A et de $7.000 \mathrm{~F}$ CFA seulement pour ceux de la catégorie C.

Dans un projet à caractère économique financé par les États-Unis d'Amérique, les responsables ont semble-t-il été choqués par cette pratique. Ils ont ainsi décidé d'égaliser le montant du per diem lors des déplacements quelles que soient les catégories de personnel. Si certains ont grincé des dents au début, les montants octroyés ont permis de faire passer la pilule. En effet, chaque déplacement donne lieu à $30.000 \mathrm{~F}$, le projet payant en plus la chambre d'hôtel! Bien que chauffeurs et cadres se retrouvent maintenant dans le même hôtel, le niveau d'indemnisation a assurément permis de faire accepter cette égalité de traitement. Dans le même ordre d'idée, des responsables d'Amnesty International nous assure ne jamais donner de per diem pour leur formation mais uniquement rembourser les frais de déplacement et prendre en charge le repas. Selon eux, cela ne pose aucun problème.

31 Face à l'inégalité d'accès à ces sources de revenus, des sentiments de jalousie et d'animosité se développent, tout comme des stratégies pour participer à des formations auxquelles a priori on ne devrait pas avoir le droit. Pour y contrecarrer, des chauffeurs d'un district sanitaire s'organisent pour partager les missions et donc les per diem et si cela n'est pas possible, «des fois tu arrives, tu lui donnes $2.000 \mathrm{~F}$ pour qu'il puisse prendre son thé ». Au Vietnam, les personnels de santé interrogés perçoivent très bien cet aspect inégalitaire et se plaignent de ne pas avoir accès à ces formations. Dichter indique que les per diem sont un des éléments qui attisent la jalousie chez les fonctionnaires à l'encontre de leurs homologues des organisations non gouvernementales (ONG). Selon l'auteur, ces sentiments conduiraient les fonctionnaires à dénigrer les accomplissements réalisés par les ONG, voire à les harceler ou les intimider. Jacquemot, quant à lui, considère que les per diem et autres incitatifs financiers contribuent à démoraliser les fonctionnaires du Ghana qui n'y ont pas accès et qui, "désabusés ", sont donc absents des processus de développement. Non seulement les sentiments de jalousie sont exacerbés mais le peu d'éthique sociale est également mise au jour. Lors d'un atelier organisé par une ONG pour discuter de la manière dont il fallait s'y prendre pour que les indigents, les plus pauvres de la société burkinabè, puissent avoir accès au système de santé malgré leur absence de moyen financier, des hauts 
responsables se sont fait remarquer. En effet, le sort des plus pauvres les intéressait moins que le montant de leur per diem « vous voulez que l'on parle de charité mais vous n'êtes pas charitable » ont-ils dit. Ces responsables de l'administration se sont même retirés de l'atelier en question car ils estimaient que le montant qui leur était attribué n'était pas suffisant. La solidarité et la justice sociale a quand même des limites!

\section{Disempowerment}

32 Le per diem est également perçu comme une forme de "disempowerment " par Phonphakdee et ses collègues. Selon les auteurs, au Cambodge, avec l'arrivée des agences d'aide internationale, les plus pauvres ont pris l'habitude d'être payés lors des divers ateliers et formations, pour prendre part à leur propre développement. Ainsi, les organisations de développement ont rendu les plus pauvres dépendants de ce type de revenus, viciant le processus d'« empowerment » des communautés. Il est donc devenu plus difficile, comme le notent Phonphakdee et al., de mobiliser les communautés sans incitatif financier. Au Burkina, une association nationale avait recours à un système de formation en cascade, regroupant des animateurs ruraux pour les former (avec per diem) dans l'espoir qu'ils s'impliquent ensuite directement avec les villageois. Mais quelques années de projets ont permis aux responsables de constater que rares étaient les animateurs qui se rendaient vraiment sur place. Aussi, a-t-il été décidé de changer de stratégie. Les formations ont été directement données aux villageois, sans leur fournir de per diem mais en organisant un simple repas communautaire. Ces derniers ont apprécié l'organisation et les activités semblent maintenant être plus efficace, nous dit un coordinateur de ce projet.

33 À travers la remise en cause de la formation comme outil de développement, la doctrine de la pérennisation des projets d'aide est également menacée. Selon Swidler et Watkins, les formations constituent l'outil de pérennisation par excellence des ONG. Reprenant le leitmotiv « quand un homme a faim, mieux vaut lui apprendre à pêcher que de lui donner un poisson ", ces formations permettent de transmettre un certain nombre de compétences aux participants qui peuvent par la suite répondre à leurs propres besoins. Les données recueillies par les auteurs montrent pourtant que les groupes communautaires soutenus par les ONG pour lutter contre le sida au Malawi n'ont pas survécu après l'arrêt des financements, et cela malgré les formations prévues pour assurer leur pérennité. La nécessité pour les individus de trouver de nouvelles sources de revenus, de nouveaux per diem, enraye la mécanique idéologique de la mobilisation communautaire et «rend [les prestataires du Sud] encore plus dépendants des planificateurs et décideurs du Nord et surtout leur fait perdre leur auto-estime » .

\section{Les effets pour la recherche en santé publique}

34 Toutes ces pratiques qui existent donc depuis fort longtemps et qui minent les interventions, de santé publique notamment, commencent aujourd'hui à s'exporter dans l'éthique de la recherche. Certes, il était temps d'encadrer un peu plus les pratiques de recherche par la mise en place des comités d'éthiques nationaux, 
comme cela se fait de plus en plus. Mais il est peut-être encore temps de réagir de manière à ce que les dérives enchâssées dans les interventions (ces dernières n'étant pas soumises à des comités d'éthique!) ne s'exportent pas dans la recherche. Déjà, sur le terrain, nous faisons face à certaines difficultés. Il s'agit de donner " l'argent du savon » à une femme après sa réponse à un questionnaire sur la mortalité maternelle pour la récompenser. Il faut donner "l'argent de la cola » au chef du village qui a bien voulu autoriser la réunion des habitants pour présenter une étude à organiser. Les habitants des villages ne sont pas dupes, ils sont parfaitement au courant des prises en charge des enquêteurs qui viennent les interroger. Aussi, prenant du temps à répondre à des questions permettant à d'autres d'écrire des rapports dont ils ne connaissent que rarement les résultats et en voient plus rarement les retombées directes, les villageois réclament de plus en plus la monnaie de leur pièce. Cela est parfois accentué par les pratiques de financement des campagnes électorales et des émoluments distribués aux villageois (ou citadins) lors des rassemblements politiques. Cela nous est arrivé récemment au Bénin, où les candidats à la députation ont distribué de tels "cadeaux", ce qui nous a posé quelques difficultés lorsque nous avons souhaité organiser des groupes de discussion avec les villageois. Or, les normes éthiques internationales insistent bien sur le fait qu'il est possible de compenser les personnes pour leur participation à la recherche, mais les montants octroyés ne doivent pas les inciter à prendre part à l'étude .

Certains comités d'éthique de la recherche en Afrique réclament maintenant des per diem pour analyser le caractère éthique des protocoles de recherche! Un comité voulait demander un montant en pourcentage du budget de la recherche tandis qu'un autre réclame $400.000 \mathrm{~F}$ CFA pour que les membres du comité puissent se réunir. Qui plus est, puisque ce tarif est appliqué pour chaque projet et que le comité est en mesure d'en étudier plusieurs dans la journée, être membre d'un tel comité devient particulièrement lucratif. Est-ce une manière de dire aux chercheurs qui ne possèdent pas de gros budgets que ce monde leur est interdit? Lorsque nous voulons organiser une réunion pour lancer une nouvelle recherche ou partager les résultats d'une recherche utile à l'action, ce qui fait maintenant partie de la responsabilité des chercheurs et est réclamé par les bailleurs de fonds, il nous faut payer des per diem aux décideurs pour qu'ils soient présents. Les connaissances deviennent-elles utiles à la prise de décision ou à l'enrichissement personnel ? Pour éviter de rentrer dans ces enjeux, nous devons lors de nos projets de recherche user de longues séances d'explication avec les parties prenantes pour bien montrer que nous faisons une étude et non pas un projet, mais la distinction est loin d'être évidente pour les villageois. Dans le monde du développement, la sémantique est parfois stratégique avons-nous vu en introduction. Les mots clefs sont essentiels, car si les gens entendent le mot projet (qu'ils associent à projet d'intervention), ils pensent rapidement qu'ils pourront en tirer quelques avantages, ce qui est moins le cas si on use du vocable "étude", avons-nous remarqué. 


\section{Conclusion} d'étude pour rendre intelligible, entre autres choses, «l'ensemble du fonctionnement (ou plutôt du dysfonctionnement) de l'État ". De plus, la gestion de la distribution des per diem pourrait être qualifiée de corruption si l'on suit certains auteurs affirmant que la corruption est constituée de deux faces, l'une publique et comprise comme illégale, et l'autre enchâssée dans les pratiques sociales et légitimée. Il n'existe évidemment pas de solutions simples à un problème aussi complexe que celui de la distribution des per diem. Et lorsque des agents sur le terrain ne souhaitent pas participer à cette mascarade, ils sont rapidement stigmatisés par leurs collègues qui se demandent bien ce qu'ils cherchent en voulant détruire cet ordre négocié . d'autres études ${ }^{21}$ au Mozambique, au Nigéria , au Mali et au Burkina Faso, est évident pour l'organisation du système de santé. Bien que les écrits soient plus rares en Asie, cela semble également poser des problèmes dans certains pays . Et il faut comprendre ici que c'est le fonctionnement par projets (qu'ils soient gérés par des africains ou des expatriés) ainsi que la dépendance à l'égard de ces derniers qui induisent en grande partie de telles pratiques. Les recherches relatives au sujet des per diem sont peu fréquentes, et on ne sait rien sur l'économie souterraine et l'apport financier de telles pratiques. C'est un sujet tabou et l'on préfère effectuer des études sur la manière dont il faut s'y prendre pour améliorer la motivation du personnel de santé ou lui payer des primes plutôt que d'évoquer cette question sensible.

Nous n'avons pas non plus ici la prétention d'avoir cerné l'ensemble des contours de ces pratiques, et l'urgence, dans un premier temps, serait de le mettre au jour et aussi, d'en mesurer l'ampleur financière. Des programmes de recherche sur cet objet sont donc nécessaires, mais il faudra en avoir le courage... et l'argent. Dans un second temps, nous pensons que les solutions ne pourront être suggérées que lors d'un processus participatif où toutes les parties prenantes seront impliquées. Car les enjeux sont tels que les décisions abruptes ne fonctionneront pas. Si l'on est par exemple capable de quantifier l'ensemble des sommes en jeu dans un pays donné, ne serait-il pas possible de demander aux bailleurs de fonds et aux gouvernements de rendre cette distribution financière officielle, équitable et transparente (ou plutôt visible) pour augmenter les salaires de tous les fonctionnaires et non pas seulement ceux qui disposent d'un réseau ou sont proches des projets? Évidemment, une telle solution d'être rapidement jetée aux oubliettes et le lecteur la trouvera quelque peu naïve. En effet, dans cet ordre négocié où l'on souhaite éviter les conflits, personne ne gagnerait à une telle transparence, ni les responsables de projets, ni les agents de santé, ni les hauts responsables, pas plus que les chercheurs ou les bailleurs de fonds. Qui osera donc mettre ce phénomène sur la place publique? Or, pour qu'une solution soit trouvée à une situation particulière, il faut que cette dernière deviennent d'abord un problème public dont les déterminants sont aussi nombreux que complexes. Malheureusement, pour le 
moment, la question des per diem n'est sur aucune table de discussion dans l'arène internationale des projets de recherche et de développement.

\section{BIBLIOGRAPHIE}

Atlani-Duault, L., \& Vidal, L. (Dir.). 2009. Anthropo-logie de l'aide humanitaire et du développement. Des pratiques aux savoirs, des savoirs aux pratiques. Paris: Armand Colin.

Bähre, E. 2005. « How to ignore corruption. reporting the shortcomings of development in South Africa », Current Anthropology, 46(1), 107-120

Berche, T. 1998. Anthropologie et santé publique en pays dogon. Paris: APAD-Karthala.

Blundo, G., \& Olivier de Sardan, J.-P. 2000. « La corruption comme terrain. Pour une approche socio-anthropologique. » In G. Blundo (dir.), Monnayer les pouvoirs. Espaces, mécanismes et représentation de la corruption. (Vol. 9, 21-46). Geneva-Paris: IUED.

Blundo, G., \& Olivier de Sardan, J.-P. 2001. « Sémiologie popu-laire de la corruption.» Politique Africaine, 83, 98-114

Bodart, C., Servais, G., Lamine, Y. M., \& Schmidt-Ehry, B. 2001. « The influence of health sector reform and external assistance in Burkina Faso. » Health Policy and Planning, 16, 74-86

Bradley, R. M., \& Karunadasa, H. I. 1989. « Community Participation in the Water Supply Sector in Sri Lanka. » The Journal of the Royal Society for the Promotion of Health, 109(4), 131-136.doi: $10.1177 / 146642408910900407$

Callon, M., Lascoumes, P., \& Barthe, Y. 2001. Agir dans un monde incertain : essai sur la démocratie technique. Paris: Seuil.

CIDR. 2006. Rapport d'étude sur la décentralisation au Nord Bénin (p. 54). Autrêches.

Comité de terminologie française de l'Ordre des comptables agréés du Québec. 2003. «Donneznous aujourd'hui notre per diem. Des équivalents français de per diem. » Terminologie comptable, 3(17), 1-2

Dambisya, Y. M. 2007. "A review of non-financial incentives for health worker retention in east and southern Africa. » In R. Loewenson (dir.), Discussion Paper (Vol. 44). Durban: Regional Network for Equity in Health in east and southern Africa.

Dichter, T. W. 1999. « Globalization and Its Effects on NGOs: Efflorescence or a Blurring of Roles and Relevance? » Nonprofit and Voluntary Sector Quarterly, 28(suppl_1), 38-58.doi: $10.1177 / 089976499773746429$

Dieleman, M., Cuong, P., Anh, L., \& Martineau, T. 2003. « Identifying factors for job motivation of rural health workers in North Viet Nam. » Human Resources for Health, 1(1), 10

Dodd, R., Hill, P., Shuey, D., \& Fernandes Antunes, A. 2009. « Paris on the Mekong: using the aid effectiveness agenda to support human resources for health in the Lao People's Democratic Republic. » Human Resources for Health, 7(1), 16 
Dovlo, D. 2005. « Wastage in the health workforce: some perspectives from African countries. » Human Resources for Health, 3(6), 1251

Dujardin, B. 2003. Politiques de santé et attentes des patients. Vers un nouveau dialogue. Paris: Karthala. Editions Charles Léopold Mayer.

Fassin, D. 2004. Des maux indicibles. Sociologie des lieux d'écoute. Paris: La Découverte.

Foster, G. M. 1987. « Bureaucratic aspects of international health agencies. » Social Science \& Medicine, 25(9), 1039-1048

Giovalucchi, F., \& Olivier de Sardan, J.-P. 2009. «Planification et gestion dans l'aide au développement : le cadre logique, outil et miroir. » Revue Tiers Monde, 198, 383-406

Gruénais, M.-E. 2005. « Les formations continues des personnels de santé en Afrique. Un marché nécessaire ? » In S. Mappa (dir.), Le savoir occidental au défi des cultures africaines: former pour changer (p. 321-350). Paris: Karthala.

Hakizimana, A. 2007 (16-11-2007). « La course au per diem, un sport national ruineux », Syfia Burundi. Repéré à http://www.syfia.info/index.php5?view=articles\&action=voir\&idArticle=4822 Heilbrunn, B. 2005. La performance, une nouvelle idéologie? : critique et enjeux. Paris: La découverte.

Israr, S. M. 2005. « Project Mentality: a threat to effective imple-mentation and sustainability of donor funded projects in Pakistan. » Journal of Pakistan Medical Association, 55(10), 457-458 Israr, S. M., Razum, O., Ndiforchu, V., \& Martiny, P. 2000. « Coping strategies of health personnel during economic crisis: A case study from Cameroon. » Tropical Medicine \& International Health, 5(4), 288-292

Jacquemot, P. 2007. « Harmonisation et appropriation de l'aide: commentaires autour de l'experience du Ghana. » Afrique contemporaine, 3-4(223), 161-191

Jaffré, Y. 2003. Chapitre 5. « Le rapport à l'autre dans des services sanitaires d'Afrique de l'Ouest (Bamako, Dakar, Niamey) » Journal International de Bioéthique 2003/1-2, 14, 101-119

Kafando, Y., \& Ridde, V. 2010. «Les ressources financières des comités de gestion du Burkina Faso peuvent améliorer l'équité d'accès au système de santé. » Cahiers Santé, 20(3), 153-161

Kamoche, K. 1997. « Competence-creation in the African public sector. » The International Journal of Public Sector Management, 10(4), 268-278

Loevinsohn, B., \& Harding, A. 2005. « Buying results? Con-tracting for health service delivery in developing countries. » Lancet, 366(9486), 676-681

McCourt, W., \& Awases, M. 2007. « Addressing the human re-sources crisis: a case study of the Namibian health service.» Human Resources for Health, 5, 1

McCoy, D., Bennett, S., Witter, S., Pond, B., Baker, B., Gow, J., McPake, B. 2008. « Salaries and incomes of health workers in sub-Saharan Africa. » The Lancet - British Edition, 371(9613), 675-681

Médecins Sans Frontières. 2007. « Health wanted. Confronting the health worker crisis to expand access to HIV/AIDS treatment: MSF experience in southern Africa. » Johannesburg: Médecins Sans Frontières.

Meessen, B., Kashala, J. P., \& Musango, L. 2007. « Output-based payment to boost staff productivity in public health centres: contracting in Kabutare district, Rwanda. » Bulletin of the World Health Organization, 85(2), 108-115.doi:S0042-96862007000200008 [pii] 
Møgedal, S., Steen, S. H., \& Mpelumbe, G. 1995. « Health sector reform and organizational issues at the local level: Lessons from selected African countries. » Journal of International Development, 7(3), 349-367

Morgan, C. J., \& Deutschmann, P. W. 2003. « An evolving model for training and education in resource-poor settings: teaching health workers to fish. » Med J Aust, 178(1), 21-25

Murray, C. J., Laakso, T., Shibuya, K., Hill, K., \& Lopez, A. D. 2007. « Can we achieve Millennium Development Goal 4 ? New analysis of country trends and forecasts of under-5 mortality to 2015 . » Lancet, 370(9592), 1040-1054

Muula, A., \& Maseko, F. 2006. « How are health professionals earning their living in Malawi? » BMC Health Services Research, 6(1), 97

Nguyen, V.-K. 2002. «Sida, ONG et la politique du témoignage en Afrique de l'Ouest. » Anthropologie et Sociétés, 26(1), 69-87

Nuffield Council on Bioethics. 2005. « The ethics of research related to healthcare in developing countries: A follow-up Discussion Paper based on the Workshop held in Cape Town ", South Africa 12-14th February 2004 (p. 128). London: Nuffield Council on Bioethics.

Olivier de Sardan, J.-P. 2000. « Dramatique déliquescence des États en Afrique. » Le Monde Diplomatique, février 2000, 12-13

-. 2005. Anthropology and Development. Understanding Contemporary Social Change. London: Zed Books.

-. 2008. «À la recherche des normes pratiques de la gouvernance réelle en Afrique », APPP Discussion Paper, 5 (http://www.institutions-africa.org/publications/discussion-papers).

-. 2011. «Promouvoir la recherche face à la consultance. Autour de l'expérience du Lasdel (Niger-Bénin). » Cahiers d'études africaines, 202-203(2-3), 511-528

Pfeiffer, J. 2003. «International NGOs and primary health care in Mozambique : the need for a new model of collaboration. » Social Science \& Medicine, 56, 725-738

Phonphakdee, S., Visal, S., \& Sauter, G. 2009. « The Urban Poor Development Fund in Cambodia: supporting local and citywide development. » Environment and Urbanization, 21(2), 569-586.doi: 10.1177/0956247809339661

Ridde, V. 2005. Building trust or buying results? Lancet, 366(9498), 1692

-. 2006. «Appréhender les conceptions locales de l'équité pour formuler les politiques publiques de santé au Burkina Faso. » Promotion \& Education, XIII(4), 252-256

-. 2008. «'The problem of the worst-off is dealt with after all other issues': The equity and health policy implementation gap in Burkina Faso. » Social Science \& Medicine, 66, 1368-1378

-. 2009a. «Le transfert de connaissances et les règles de fonctionnement du système universitaire: besoin de changementsi. » Global Health Promotion, 16(3), 70-72

-. 2009b. «Policy implementation in an African State : an extension of the Kingdon's multiplestreams approach. » Public Administration, 87(4), 938-954

-. 2010. «Per diems undermine health interventions, systems and research in Africa: burying our heads in the sand. » Tropical Medicine \& International Health, Jul 28. doi: 10.1111/j. 1365-3156.2010.02607.x. 
Ridde, V., Zerbo, R., Yaogo, M., Faye, A., \& Samb, O. 2012. Les deux solitudes : les systèmes de recherche et d'organisation des soins de santé au Burkina Faso à travers l'histoire du paiement des soins par les patients. Document de travail. Montréal: CRCHUM/CNRST/AFRICSanté.

Rochefort, D. A., \& Cobb, R. W. (1994). « Problem definition : an emerging perspective.» In D. A. Rochefort \& R. W. Cobb (dir.), The politics of problem definition. shaping the policy agenda (p. 1-31). Lawrence, Kansas: University Press of Kansas.

Roenen, C., Ferrinho, P., Van Dormael, M., Conceição, M. C., \& Van Lerberghe, W. 1997. « How African doctors make ends meet: an exploration. » Tropical Medicine \& International Health, 2(2), 127-135

Schaffer, B. B. 1974. « Introduction: the ideas and institutions of training. » In B. Schaffer (dir.), Administrative training and development. A comparative study of East Africa, Zambia, Pakistan, and India. New York Praeger.

Sedes. 2008. Rapport provisoire sur l'impact des mesures d'incitation financière de 2006 (p. 71). Niamey: Ministère de la Santé Publique.

Smith, D. J. 2003. « Patronage, per diems and the "Workshop mentality": the practice of family planning programs in Southeastern Nigeria. » World Development, 31(4), 703-715

Strauss, A. L. 1992. La trame de la négociation : sociologie qualitative et interactionnisme. Paris: LHarmattan.

Swidler, A., \& Watkins, S. C. 2009. «'Teach a man to fish': The sustainability doctrine and its social consequences. » World Development, 37(7), 1182-1196

Turner, M. M. 1989. 'Trainingism' revisited in Papua New Guinea. Public Administration and Development, 9(1), 17-28.

Vidal, L., Fall, A. S., \& D., G. (dir.). 2005. Les Professionnels de Santé en Afrique de L'ouest: entre savoirs et pratiques : paludisme, tuberculose et prévention au Sénégal et en Côte d'ivoire. Paris: LHarmattan.

Witter, S., Kusi, A., \& Aikins, M. 2007. « Working practices and incomes of health workers: evidence from an evaluation of a delivery fee exemption scheme in Ghana. » Human Resources for Health, 5, 2-2.

\section{NOTES}

1. Qualificatif emprunté à De Gaulejac V. 2005, tel que cité par .

2. Ce texte est tiré d'une communication effectuée lors du colloque de l'APAD en janvier 2010. Une version (très) courte a été publiée en anglais . Je tiens à remercier Fatoumata Hane, Oumar Mallé Samb, Fatoumata Ouattara-Traoré et Sten Hagberg pour leurs commentaires constructifs concernant ce texte. Valéry Ridde est Nouveau Chercheur des Instituts de Recherche en Santé (IRSC) du Canada.

3. Sur le même sujet, voir l'article consacré à la sémiologie populaire de la corruption .

4. Medicine Digest, vol 15 (5), May 1989, p.55.

5. Nous mettons ici le terme « quasiment » car deux auteurs de chapitres l'évoquent furtivement sans en faire un objet d'étude et tout aussi rapidement le chapitre conclusif suggère même, dans un exemple, de donner des primes aux agents de santé qui seraient surchargés dans leurs tâches professionnelles. Paradoxalement, alors que de l'avis des principaux acteurs de terrain rencontrés c'est bien dans le secteur de la santé que cette pratique est la plus prégnante («la 
santé c'est grave » nous dit un interlocuteur), le chapitre consacré à la santé dans cet ouvrage ne dit mot de ces perdiem.

6. Pour des raisons éthiques, le minimum d'informations est fourni au lecteur concernant l'origine des données qui n'en restent pas moins empiriques.

7. Nous tenons à remercier Emilie Robert pour son repérage et analyse des quelques rares écrits scientifiques sur le sujet.

8. Par simplification sémantique nous parlons ici d'efficacité mais il faudrait plutôt employer le terme de «mise en œuvre " car la production d'effets tangibles, de changements certains pour les populations, restent encore à prouver. L'efficacité dans ce type de contexte se réfère donc plus à la mise en œuvre d'activité qu'à l'évaluation des effets que ces dernières ont produit. Par exemple, dans nombre de plans de district sanitaire, on voit maintenant fleurir des indicateurs symptomatiques de cette approche car pour rendre des comptes à leurs bailleurs fonds, les responsables calculent maintenant le "taux d'exécution physique " soit le nombre d'activités réalisées par rapport au nombre prévues... selon cette fameuse maladie de la quantophrénie ; ce que ces activités ont changé pour les populations est une autre histoire.

9. Sur le sujet de la consultation en Afrique

10. La définition même des projets de développement consacrée dans les décrets présidentiels de 2007 au Burkina est éloquente de l'absence de volonté de changer une situation problématique car un projet est défini comme «toutes actions d'investissement publics ou de coopération technique, exécutées pendant une période déterminée, dans le but de produire des biens et des services; réaliser des infrastructures socio-économiques; renforcer les capacités institutionnelles de l'administration centrale, déconcentrée et locale, de la société civile; renforcer les capacités et compétences des ressources humaines » (c'est nous qui soulignons)

11. " les autorités sanitaires nous ont suggéré de suivre les barèmes du PADS ", dit un responsable de projet dans le domaine de la santé. Ceci montre, d'une part, que tous les fonctionnaires ne semblent pas en phase avec les décrets présidentiels, et, d'autre part, que certains choisissent aussi la norme la plus avantageuse financièrement car les barèmes du PADS sont pour certaines catégories plus élevés que ceux des décrets.

12. On ne parlera pas ici de la complexité des primes qui sont attribuées aux professionnels de santé, ces dernières étant souvent plus importante en valeur absolu que le salaire de base dans de nombreux pays. Au Niger par exemple, les avantages alloués aux médecins, pharmaciens et chirurgiens ne comportent pas moins de huit primes différentes (sujétion, fonction, motivation, risque, logement, téléphone, roulage, garde). Pour un médecin généraliste, ces primes équivalent de 2,5 à 3,2 fois le salaire de base, ce qui n'empêche pas qu'en 2008 il reste encore plus de médecins dans la capitale $(\mathrm{n}=158)$ qu'ailleurs dans le pays $(\mathrm{n}=134)$.

13. Notons cependant que pendant des années, la centrale d'achat des médicaments (CAMEG) du Burkina Faso distribuait des ristournes sur les achats aux responsables des commandes dans les districts. Les sommes étaient importantes, les médecins chefs n'étaient pas au courant, et les chèques étaient délivrés à titre personnel et non pour le district sanitaire.

14. Il faut bien rappeler que ces agents sont des salariés, certes faiblement rémunérés (au minimum d'environ 60.000 F FCFA par mois officiel et sans perdiem), mais au Burkina Faso, 44\% de la population dépensent moins de $100.000 \mathrm{~F}$ par an et par habitant, soit moins de $250 \mathrm{~F}$ par jour.

15. Ce qui montre bien que tout le monde est partie prenante de ce processus et qu'il ne s'agit pas pour nous de nous exclure de cette pratique mais de tenter de mieux la révéler et d'en comprendre les mécanismes.

16. D'un autre côté, Dujardin dit « comprendre l'importance et la fréquence » des pratiques malhonnêtes compte tenu de «l'écart entre le salaire officiel et les besoins financiers réels des professionnels de la santé pour être honnêtes dans leur «métier » de chef de famille, c'est-à-dire être capables d'élever dignement leurs enfants» (p.140). Dignité et corruption vont-ils de paires? 
17. On se rappellera que le secteur de la santé a été classé 2ème secteur le plus corrompu après la Douane au Burkina Faso en 2003 par le REN-LAC.

18. Dans l'analyse de Schaffer reprise par Turner dans son étude sur la Papouasie NouvelleGuinée , le «trainingism » s'applique à l'origine aux instituts de formation administrative créés sous la décolonisation pour former les classes dirigeantes des pays nouvellement indépendants. Selon l'auteur, ces instituts ont érigé la formation comme la solution miracle, voire un véritable acte de foi pour reprendre les mots de Kamoche, pour résoudre les problèmes d'ordre organisationnel, et ainsi assurer la pérennité de leurs activités.

19. Pour avoir un ordre d'idée, la politique de subvention des accouchements à l'échelle nationale afin de favoriser une prise charge médicale coûte moins de 2 milliards par an à l'État

20. Adapté d'un décret de 1960, montrant que la question est ancienne

21. Pour l'Éthiopie voir http://www.coady.stfx.ca/coady/features/0810/perdiem

\section{RÉSUMÉS}

La pratique des per diem dans les projets est largement connue de tous les acteurs concernés par l'aide publique au développement. En plus de leur salaire, des fonctionnaires touchent des primes fournies par des responsables de projets pour se réunir, pour participer à des ateliers et des formations ou pour réaliser des activités qui sont pourtant prévues dans leurs tâches officielles. D'un autre côté, des experts internationaux reçoivent des frais de mission qui dépassent souvent largement le montant des dépenses nécessaires pour vivre le temps de leur mission. Si ces pratiques sont connues, leur description et discussion sont inversement proportionnelles à leur influence dans la mise en œuvre des interventions. Nous proposons dans cet article une esquisse d'anthropologie de cette pratique. Son histoire et ses origines multiples sont reconstituées, nous en décrivons les diverses facettes, leurs causes et conséquences afin d'en proposer une typologie. Nous tentons, enfin, d'analyser en quoi les per diem sont révélateurs des effets pervers de l'aide, de la transformation du rôle de l'État, et des changements de la fonction publique en Afrique.

The practice of per diems in projects is widely known by all actors involved in development aid. In addition to their salary civil servants get allowances from project managers for meetings, participation in workshops and trainings or to execute professional tasks. On the other hand, international experts receive daily allowances well beyond the living expenses in the country they are visiting. These practices are well-known, and yet they are seldom described or discussed, especially considering their important influence on the implementation of development interventions. In this article we sketch out an anthropology of this practice. The retracing of its history and description of its diverse expressions, its causes and consequences, will lead to the suggestion of a typology. Finally, we set out to analyse in what ways per diems are revelatory of the perverse effects of aid, of the transformation of the state and of changes in the public service in Africa. 